African Journal for Physical, Health Education, Recreation and Dance (AJPHERD)

Vol. 15, No. 3 (September) 2009, pp. 493-505.

\title{
Effect of visual cues and practice on decision making of touch rugby players
}

\author{
RUSSELL I. CLARKE ${ }^{1}$, JANINE D. BRUMMER ${ }^{1}$, \\ DARLENE A. KLUKA ${ }^{2}$ AND ANNELIESE E. GOSLIN ${ }^{1}$ \\ ${ }^{1}$ Department of Biokinetics, Sport and Leisure, Sciences, Centre for Leisure Studies \\ University of Pretoria, South Africa and ${ }^{2}$ Department of Sport and Exercise Sciences \\ Barry University, Miami Shores, Florida USA; Email: goslin@sport.up.ac.za
}

(Received: 2 December 2008 Revision Accepted: 8 July 2009)

\begin{abstract}
Researchers have reported that appropriate decision making involves an understanding of choices between options and performing appropriate actions. The ability to identify visual cues that improve decision making and performance is beneficial for sport; however, after a thorough perusal, virtually no published information is available on visual training, decision making and touch rugby. The aim of this study was to determine the effects of visual cues and practice on decision making of touch rugby players. A convenience sample of 18 South African provincial touch rugby players were selected and divided randomly into three groups. Groups were required to analyze touch rugby-specific situations from video clips and determine the best decision for each one. Group 1 participated in a physical training session based on defense in touch rugby and a visual training session which involved analyzing defense patterns. Group 2 only participated in a physical training session based on defense. Group 3 acted as a control and was not involved in any additional training sessions. Results from a KruskalWallis test indicated that there was no significant difference $(\mathrm{p} \leq 0.10)$ between the pretest of the three groups. There was, however, a significant difference $(p \leq 0.10)$ in the post-test among the three groups in terms of body positioning, field positioning, response time and verbal communication with reference to decision making in touch rugby. It is recommended that additional research that replicates the research design be performed in order to construct more definitive interventions for decision making in touch rugby.
\end{abstract}

Key words: Visual cues, decision making, touch rugby, video observation.

\section{Introduction}

Visual training and decision making have been under much scrutiny for many years in order to determine whether they actually affect an athlete's judgment and choices made during competition and thus affect performance. Judgment and decision making investigations can be traced back to the 1940s and included decision and game theory involving psycho-social aspects. In all studies, however, the main focus of decision making remained in the understanding of choices between options (Editorial, 2006). Visual cues, strategies, and awareness form a vital part of actions that result from specific decisions. Observation of movements and actions in sport place an increased demand on an athlete's vision, as vision involves many subtle and sophisticated links between the eyes, the brain and voluntary muscles of the body (Verrell, 2004). 
Visual perception and decision making are vital to appropriate actions (Kluka, 1999). Visual cues or strategies form a basis for the decision making process (Vickers, 2007). Decision making is an outcome of mental processes leading to the selection of actions. Determinants of these final actions range from environmental, physical, physiological and knowledge-based cues (Tennenbaum, 2003).

Environmentally- and physically-based cues relate to body positioning, field positioning and the execution of actions. These form part of the psychologically-based cues which form working and long term memory in making action-related decisions. Knowledge-based cues are experiences which are attained from previous decision making situations.

Levels of visual awareness involved in team sports are greater than individual sports and involve great contribution to decisions being made in context, thereby indirectly affecting performance. Investigators involved with youth soccer players (Vaeyens, Lenoir, Williams \& Philippaerts, 2007) identified each soccer player using visual cues in decision making. It is, however, important that the entire team and opponents are integrated into decisions and not only a single fixation on an individual in possession of the ball. Team sports also require athletes to integrate visual cues and actions in order for decision making to be effective (Rulence-Paques, Fruchart, Dru \& Mullet, 2005).

In team sports, it is important to choose the appropriate action at the right moment. Researchers (Grehaigne, Godbout \& Bouthier, 2001) focusing on team sports have determined that although experts make more accurate decisions based on earlier acquisition of information, they tend to have more speed and less accuracy. This can, however, be improved by creating learning environments that include observation and verbalization which bring about more critical thinking and develop strategic and tactical knowledge (Raad, Masters \& Maxwell, 2005). Calder (1993) substantiated the construct discussed by Raad and colleagues (2005) by investigating the effect of sportspecific visual cue skills acquisition in female field hockey players.

An intervention strategy of sport-specific anticipatory visual cue acquisition and decision making in addition to sport-specific physical practice significantly enhanced players' abilities to perform field hockey-specific skills for game performance success.

Decision making is also dependent on the "how" and "what" of decisions made. The "how" involves the technical aspects or techniques, and the "what" includes the tactical aspects of what an individual or team wants to achieve. Results of studies by Raad and colleagues (2005) have shown that improvements on the "how" and the 
"what" of decisions can be achieved through a combination of technical and tactical experiences in early season training programmes.

Elite levels of sporting individuals acquire contrasting visual cues to attain performance and minimize visual skill errors. Elite sporting individuals have developed a greater expertise, gaining the ability to allocate visual spatial attention and both focal and peripheral vision (Ripoll, Kerlirzin, Stein \& Reine, 1995; Kluka, Love, Sanet, Stroops \& Schneider, 1995).

As variations in psychological, physical and environmental characteristics play roles in the split-second decision making process, decisions made can occur by chance. In a study conducted by Vickers (2007), however, the ability of individuals to identify and facilitate visual perception and actions has shown to enhance performance. In another study of softball batters, anticipatory visual cues, and hitting performance, Kellers (1988) determined that pitch selection was significantly improved by video pitch analysis and hitting practice, rather than hitting practice alone.

Research on the methodology and effects of sport-specific visual training on decision making in sport is somewhat limited. Some researchers have shown that visual training can be beneficial for baseball, cricket, football, field hockey and volleyball (Calder \& Kluka, 2009; Douglass-Thom, 2007; Kluka, Love, Kuhlman, Hammack \& Wesson, 1994).

If decisions being made by athletes have positive effects on performance, it is logical for visual cues and the practice thereof to be further researched. There is limited information regarding research on visual training and touch rugby players. Through empirical knowledge about the game, decision making forms a vital part in making appropriate actions, attaining an effective position, and improving total reaction time to decisions made during this high passing game.

The aims of this investigation were to:

(1) determine what effect visual cues practice had on decision making of touch rugby players;

(2) determine if practising cues improved decisions made by touch rugby players;

(3) contribute to the body of knowledge on the effect of visual cues and decision making in touch rugby performance.

\section{Methodology}

The study of visual cues and practice in decision making was an experimental study which involved athletes who were randomly assigned to one of three groups. A checklist was constructed to obtain what decisions were made by athletes involved. 
Three elite South African touch rugby coaches analyzed a video game between Australia and New Zealand to establish clips involving touch rugby moves and tactics on defense. Inter-rater reliability was found to be 0.96 . Intra-rater reliability was established at 0.98. Each video clip was further analyzed to determine and rank the most effective to the least effective decisions according to visual cues that could be made for each of the eight situations. Each video clip had four possible decisions for each situation. The checklist consisted of four randomly placed multiple choice decisions. The checklist was in the form of a booklet, each containing a set of four possible answers for each clip. This was repeated on separate pages, three times per clip.

During video analysis and video training in a lecture room, the video was shown through a data projector to ensure optimal vision. Athletes were also limited to sitting in a specific section of the room to ensure consistency of peripheral vision utilized in viewing.

After obtaining ethical clearance through the Institutional Review Board at the University of Pretoria, South Africa, each group received the same pre- and post-test procedures. Two of the groups received physical visual training; one group received additional visual training. The third was used as a control, receiving no extra physical or visual training. Table 1 represents a graphic of the study design.

Table 1: Study Design

\begin{tabular}{|l|l|l|l|}
\hline Group 1 & Pre-test & $\begin{array}{l}\text { Training drills and visual training } \\
\text { session }\end{array}$ & Post-test \\
\hline Group 2 & Pre-test & Training drills & Post-test \\
\hline Group 3 (control) & Pre-test & N/A & Post-test \\
\hline
\end{tabular}

Researchers (Abernethy \& Bruce, 1991) have indicated that decisions made by expert elite players differ from less experienced ones. As this aspect may influence results in decisions made, it is essential that athletes in this study were determined as homogeneous in touch rugby performance. The tests were administered to 18 male provincial players in South Africa's Gauteng Province. Each player had at least two years of provincial competitive experience and who were aged between 20 and 30 years.

Touch rugby, relatively new to South Africa, has a limited number of players. Therefore, the athlete selection was based on convenience sampling. Those selected were randomly divided into three groups, 6 athletes per group.

The study was performed on one day for test procedures to eliminate the effect on visual information being lost by athletes. Testing was performed at the University of Pretoria, South Africa, video training was conducted in a large lecture room, and 
physical training on a regulation rugby field. Upon arrival, the athletes were briefed in and completed all necessary documentation. Athletes also took a Snellen Eye test to establish their static vision. Athletes were required to take part in the pre-and posttests and a training session (if in Group 1 or 2).

Pre-Test: Athletes were required to watch and analyze selected video clips from an international touch rugby match played in 2007 between Australia and New Zealand. Using the checklist, the athletes selected which of the four possible decisions they would have performed in that specific situation.

Each video clip was shown three times with a 15-s gap in between in order to allow realistic time to respond after viewing each clip. Each athlete had his own checklist booklet. The possible multiple choice answers for every clip were only allowed to be viewed by the athlete after the first viewing of each clip. This allowed athletes to give their own interpretation of each clip and were thus not assisted by which aspects to concentrate on. Each clip was played 3 consecutive times which allowed the athlete to change the answer if necessary (Kluka, 1999).

Training Session: Groups 1 and 2 participated in physical training sessions. The drills performed were obtained from an international level South African coach and were conducted by the Northern Gauteng provincial coach. Drills were based on touch rugby defense related to visual awareness and training of the athletes.

Visual Training Session: Following the training session, athletes from Group 1 participated in an additional video session. Video footage consisted of a series of club matches played in 2007 obtained from the South African Touch Rugby Association. Situations on the video were analyzed interactively by the group and the Northern Gauteng provincial coach to establish the most effective decisions in context. Video simulations were watched several times with open discussions and no time limits.

Post-test: After all training session, the three groups were required to watch and analyze the same 8 video clips under identical conditions and context as the pretest. The exact procedures were followed as in the pretest, and the athletes responded with the most effective decision they would perform in each situation.

Data Analysis: The Kruskal-Wallis test was used to determine if there was a significant difference between the three groups on pre- and post-test variables. To determine if significant differences existed within each group, the Wilcoxon Signed test was used. Descriptive statistics of each group, specifically the mean and standard deviation, were also determined during data interpretation. 


\section{Results and Discussion}

A significance criterion of $\mathrm{p} \leq 0.10$ was used for all results reported. Numerical values were allocated to each answer, the lowest number representing the best possible answer; the highest number represented the worst possible answer. The KruskalWallis test was used to determine any significant differences between the three groups on all pre- and post-test variables. By using the Kruskal-Wallis, it was found that there was no statistically significant difference between the three groups in the pretest, except in clip 4c, which was based on field positioning (Group 1 (2.67), Group 3 (2.17), Group 2 (1.17). This would make any significant differences in clip 4 void. It was essential to eliminate this clip as the athletes were required to be of equal standards in order for the study to have comparable results; therefore, any improvement was likely due to the intervention used in the study.

There was, however, a significant difference in the post-test scores between the three groups on the following clips:

Clip 2 involved the decisions made on athlete body position, mainly shoulder orientation, in the midfield position during defense. In clip 2a, Group 1 had the highest value (2.83), followed by Group 3 (2.50), with Group 2 (1.50) having the lowest value.

Clip 3 required the athlete to make decisions regarding field positioning during a defensive pattern, relevant to teammate positioning. It was found that in clip 3a, Group 2 had the highest value (3.17), followed by Group 3 (2.17), and Group 1 (2.00) had the lowest value.

Clip 6 required the athlete to make a decision regarding the change in defensive pattern which involved visual cues given by the attacking team. In clip 6a, Group 3 had the highest value (3.17), followed by Group 1 (1.83), Group 2 (1.50) with the lowest value. In clip 6b, Group 3 had the highest value (2.83), followed by Group 1 (2.17), and Group 2 with the lowest value (1.00). Clip 8 involved verbal communication between defensive players relative to decision making.

In clip 8c, Group 2 had the highest score (2.67), followed by Group 3 (1.83), while Group 1 (1.33) had the lowest score. Figure 1 represents the information above in graphic form.

The Wilcoxon Signed Ranks test was used to establish whether any statistically significant differences existed within each group on all variables measured, thereby testing differences between groups in pre- and post-tests. 


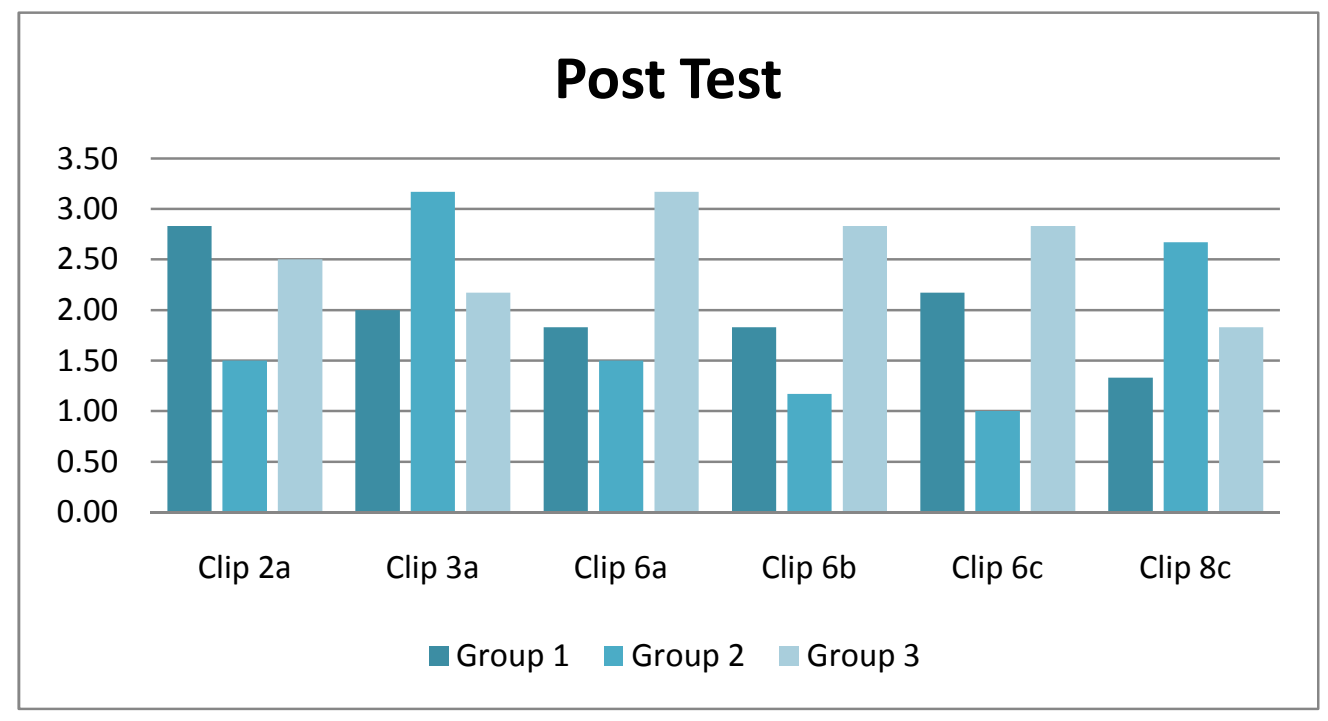

Figure 1. Significant differences among groups in post-test.

Group 1: Clip 2 involved decision making of body positioning during defense. Clip $2 \mathrm{~b}$ 's pre-test value (3.00) was significantly higher than post-test value (2.50); therefore, the intervention significantly decreased post-test value, indicating an improvement in decisions made by defensive players' shoulder positioning with regard to opponents. A similar finding was corroborated by Kellers (1988) relative to softball pitch decision making. Although the two sports (softball and touch rugby) differ in action technique, they display similarities in anticipatory visual cue sets and coordinated effort. Both involve an object approaching on a predictable path, anticipatory visual cue input and appropriate decision making relative to actions needed for performance success.

Clip 5 required the athlete to make decisions regarding field positioning during a defensive pattern. Clip 5b's pre-test value (2.67) was significantly greater than the post-test value (1.83); therefore, the intervention significantly decreased the post-test value, indicating an improvement in decisions made by two midfield athletes regarding field positioning and retreat after a touch just outside the in-goal area.

Clip 7 required athletes to make decisions about field positioning during a defensive pattern. Clip 7c's pre-test value (3.00) was significantly higher than post-test value (2.50), thereby indicating a significant decrease in the post-test value. This illustrated an improvement in decisions made in the midfield position related to the opposition.

Clip 8 involves verbal communication between defensive players with regard to decision making. Clip 8a's pre-test value (2.33) was significantly higher than the posttest value (1.50), thus showing an improvement in decisions regarding verbal 
communication between defensive players. Figure 2 is a graphic representation of differences.

Figure 2 displays an improvement in decision making as a result of the intervention used in the study. This improvement could be the result of the athlete's enhanced understanding of decision making aspects relative to field position of the attacking team, body positioning and orientation, and verbal communication between teammates. This improvement may also be a result of the combination of the intervention and athletes' actual performances in different body orientations, communication and field positioning activities with teammates relative to defensive patterns and being able to identify and analyze visually in each visual training session. These findings appear similar to those of an earlier study conducted by Calder (1993). Field hockey and touch rugby both played primarily outdoors on a similarly sized field and under outdoor lighting conditions, appear to involve spatially similar attack and defensive field positioning, particularly by midfielders.

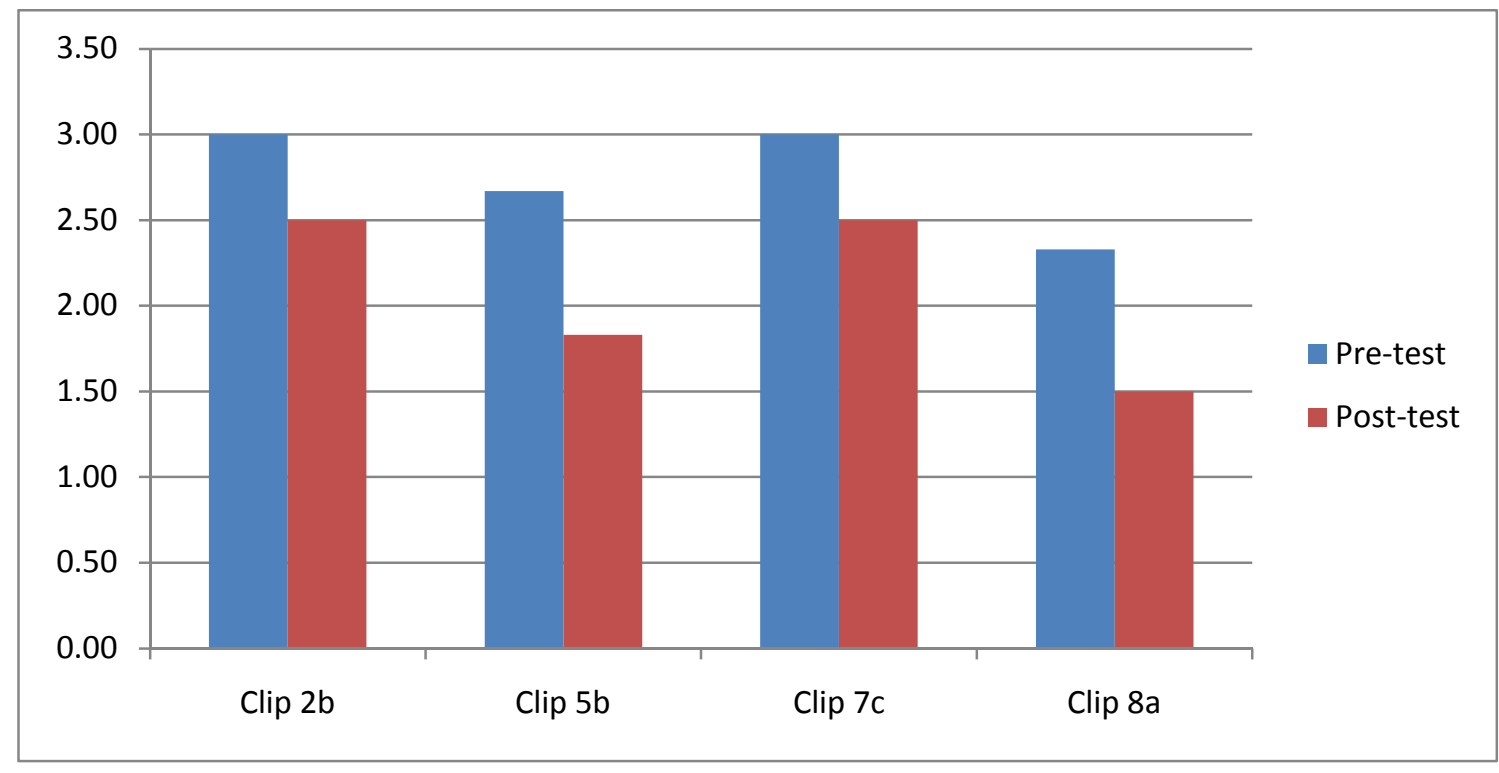

Figure 2. Significant differences between pre and post-test in Group 1

Calder concluded that sport-specific visual skills training in the context of the game significantly enhanced the performance of sport-specific skills.

Group 2: Clip 2 involved decision making of body positioning during defense. Clip 2a's pre-test value (2.33) was significantly higher than the post-test value (1.50). Clip 2 b's pre-test value (2.50) was significantly higher than the post-test value (2.00); therefore, the intervention significantly decreased the post-test value, indicating an 
improvement in both clips regarding decisions made by defensive players and shoulder positioning relative to opponents.

Clip 6 required athletes to make a decision to change a defensive pattern involving visual cues presented by the attacking team. Clip 6b's pre-test value (2.33) was significantly higher than the post-test value (1.17); therefore, the intervention significantly decreased the post-test value, indicating an improvement in decisions made when viewing certain visual cues from the attacking opposition to alter defensive structures and strategies. Although there was also a significant difference found in clips $1 \mathrm{~b}, 3 \mathrm{a}$, and $3 \mathrm{c}$ (based on field positioning), there was an increase rather than a decrease in the post-test values, indicating a decreased performance in decision making: clip $1 \mathrm{~b}$ pre-test (1.50), post-test (2.83); clip 3a pre-test (2.00), post-test (3.17); clip 3c (pre-test (1.50), post-test (2.33). Figure 3 presents significant differences between pre- and post-tests in Group 2.

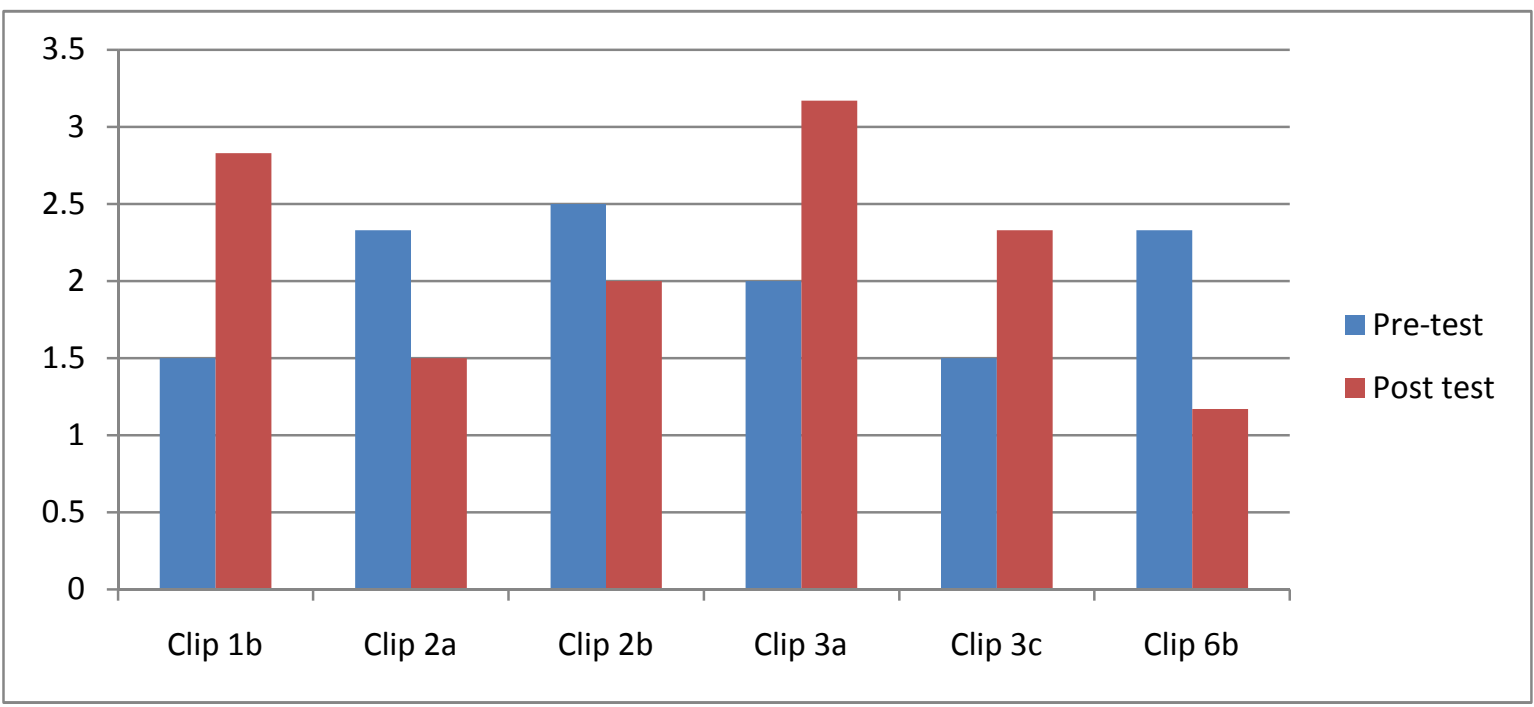

Figure 3. Significant differences between pre and post-tests in Group 2

The results achieved by Group 2 thus show that athletes improved in their decision making relative to body orientation and visual cues received from the attacking side, but did little for field positioning. This may be due to the athletes' inabilities to see where he should be in relation to teammates and attacking players, as showed in the visual training session performed by Group 1. The decrease in Group 2's performance may also be attributed to the group's loss of interest, as each had an uninformative waiting period while Group 1 took part in the visual training before the post-test.

Group 3: This was the control group and showed no significant differences in any of the clips, as they were not involved in any of the additional sessions. No improvement was recorded in decision making performance. These are similar findings to 
investigations conducted by Calder (1993), Grehaigne and colleagues (2001), Raad and colleagues (2005), and Vickers (2007).

The above results showed a significantly greater improvement in Group 1's performance, followed by Group 2, with no improvement in Group 3. The results found in this study show similar findings by Vickers (2007) that the ability of individuals to identify and facilitate visual perceptions and reactions can enhance performance. The improvements found in Groups 1 and 2 indicate that athletes were able to enhance their abilities to identify and process information presented by sportspecific visual cues presented in context using video observation, which can lead to enhanced performance in decision making. It is, then, feasible that sport-specific visual cue enhancement programs using video observation, when supplemented by sport-specific physical practice, can improve sport-specific performance relative to decision making.

During team sports, athletes must process information nearly simultaneously, not only on the opponent's actions and organization but also their teammates' actions and organization (Rulence-Paques, et al., 2005). Team sports require athletes to integrate visual cues and decision making in order to alter actions in order to perform effectively relative to time, space, force and flow. This is clear in the results found in several of the above-mentioned clips, as athletes were required to consider teammate or opponent positioning and defensive structure to make effective decisions. This is illustrated in Table 2.

\section{Conclusion and Recommendations}

This study focused on the effect of visual cues and practice on decision making and whether or not practice of visual cues improves decisions made by touch rugby players. It is clear that the group who participated in touch rugby-specific visual cue enhancement and touch rugby-specific practice sessions displayed a greater improvement in decisions made involving touch rugby-specific moves and tactics based on field positioning, body positioning and verbal communication. The additional visual cue practice session allowed athletes to better understand, not only the physical aspects involved with decision making in touch rugby, but also the visual identification and process of decisions to be made.

The group that participated in only the physical practice session showed less improvement than the group with both visual cue and physical practice. Although they understood the physical aspects of performance related to decision making, they were unable to effectively identify the visual aspects needed for effective decision making. It can be concluded that sport-specific visual cues and sport-specific physical practice can result in an improvement in sport-specific performance. 
Table 2. Descriptive values of pre and post-test in groups.

\begin{tabular}{|c|c|c|c|c|c|c|}
\hline Clips & Group 1 Pre-test & Group 1 Post-test & Group 2 Pre- test & Group 2 Post-test & Group 3 Pre-test & Group 3 Post-test \\
\hline Clip 1 a & 2.50 & 2.17 & 3.00 & 2.67 & 2.33 & 2.83 \\
\hline Clip 1 b & 1.83 & 2.17 & 1.50 & 2.83 & 1.83 & 2.50 \\
\hline Clip 1 c & 2.50 & 2.00 & 2.33 & 2.33 & 1.67 & 2.50 \\
\hline Clip 2 a & 3.50 & 2.83 & 2.33 & 1.50 & 2.83 & 2.50 \\
\hline Clip 2 b & 3.00 & 2.50 & 2.50 & 2.00 & 2.50 & 2.17 \\
\hline Clip 2 c & 2.67 & 2.83 & 2.67 & 2.33 & 2.33 & 2.50 \\
\hline Clip $3 \mathrm{a}$ & 3.00 & 2.00 & 2.00 & 3.17 & 3.00 & 2.17 \\
\hline Clip 3 b & 2.17 & 3.00 & 2.17 & 2.50 & 2.50 & 2.00 \\
\hline Clip 3 c & 2.17 & 2.50 & 1.50 & 2.33 & 2.33 & 2.00 \\
\hline Clip 4 a & 2.50 & 1.83 & 1.50 & 1.83 & 2.33 & 2.33 \\
\hline Clip 4 b & 2.33 & 2.33 & 1.17 & 2.50 & 2.00 & 2.67 \\
\hline Clip 4 c & 2.67 & 2.67 & 1.17 & 2.00 & 2.17 & 2.67 \\
\hline Clip 5 a & 1.67 & 2.33 & 2.00 & 1.83 & 2.33 & 2.00 \\
\hline Clip 5 b & 2.67 & 1.83 & 2.33 & 1.83 & 2.00 & 2.17 \\
\hline Clip 5 c & 2.17 & 1.83 & 2.33 & 2.50 & 1.67 & 1.83 \\
\hline Clip $6 \mathrm{a}$ & 2.67 & 1.83 & 2.67 & 1.50 & 2.67 & 3.17 \\
\hline Clip 6 b & 3.17 & 1.83 & 2.33 & 1.17 & 3.00 & 2.83 \\
\hline Clip $6 \mathrm{c}$ & 2.67 & 2.17 & 1.83 & 1.00 & 3.33 & 2.83 \\
\hline Clip $7 \mathrm{a}$ & 2.33 & 2.67 & 2.83 & 2.33 & 2.67 & 2.67 \\
\hline Clip 7 b & 3.00 & 2.67 & 2.83 & 2.50 & 2.67 & 3.00 \\
\hline Clip 7 c & 3.00 & 2.50 & 2.83 & 2.50 & 2.67 & 3.00 \\
\hline Clip $8 \mathrm{a}$ & 2.33 & 1.50 & 2.17 & 2.33 & 2.33 & 2.00 \\
\hline Clip 8 b & 2.00 & 1.33 & 2.00 & 2.00 & 1.50 & 1.83 \\
\hline Clip $8 \mathrm{c}$ & 1.50 & 1.33 & 2.17 & 2.67 & 1.50 & 1.83 \\
\hline Average: & 2.50 & 2.19 & 2.17 & 2.17 & 2.34 & 2.42 \\
\hline
\end{tabular}

It is recommended that valid and reliable sport-specific visual cue enhancement programs using video observation, when supplemented by sport-specific physical practice, be used to improve sport-specific decision making in order to enhance physical performance.

\section{Acknowledgements}

The authors are grateful to the following people for their assistance during the study: Sean Smith and Richard Mullins (for formation of the checklist); Northern Gauteng Touch Rugby Association (for use of touch rugby players); coaches (for their 
assistance in formation of the checklist and obtaining relevant visual training data) and Marlize Alexander (for assistance in statistical analysis of data).

\section{References}

Abernethy, B. \& Bruce, J. (1991). Visual search strategies and decision making in sport. International Journal of Sport Psychology, 22(3-4), 189-210.

Calder, S. L. (1993). Effects of visual skills awareness training and field hockey practice. University of Cape Town, South Africa: Unpublished doctoral thesis.

Calder, S. L. \& Kluka, D. A. (2009). The efficacy of the EyeThinkSport training programme on selected South African high school cricketers. African Journal of Physical, Health Education, Recreation and Dance, 15(1), 44-61.

Douglass - Thom, K. (2007). High performance vision. Experience Life Magazine, 24-27.

Editorial (2006). Judgement and decision making in sport and exercise: Rediscovery and new visions. Psychology of Sport and Exercise, 7, 519-524.

Grehaigne, J.F., Godbout, P. \& Bouthier, D. (2001). The teaching and learning of decision making in team sports. Quest, 55, 59-76.

Kellers, P. (1988). Videography use in estimation of softball pitch accuracy. Unpublished doctoral dissertation: University of Virginia.

Kluka, D. A. (1999). Motor behaviour: From learning to performance. New York: Thompson Learning Group.

Kluka, D., Love, P., Kuhlman, J., Hammack, G. \& Wesson, M. (1996). Effects of a visual skills training program upon selected intercollegiate women volleyball athletes. International Journal of Sports Vision, 3(1), 22-25.

Kluka, D., Love, P., Sanet, R., Hillier, C., Stroops, S. \& Schneider, H. (1995). Contrast sensitivity function profiling by sport and physical ability level. International Journal of Sports Vision, 2(1), 5-16.

Nettleton, B. (2001). Decision making in sport. Sports Coach, 24(2), 32 - 33.

Raad, M., Masters, R. \& Maxwell, J. (2005). Improving the how and what decisions of elite table tennis player. Human Movement Science, 24, 326-344.

Ripoll, H., Kerlirzin, Y., Stein, J. \& Reine, B. (1995). Analysis of information processing, decision making and visual strategies in complex problem solving sport situations. Human Movement Science, 14, 325-349.

Rulence - Paques, P., Fruchart, E., Dru, V. \& Mullet, E. (2005). Decision making in soccer game: a developmental perspective. Revue Europeenne de Psychologie Appliqué, 55, 131-136.

Tennenbaum, G. (2003). An integrated approach to decision making. In J. Starkes \& K. Erricsson (Eds.), Expert Performances in Sport (pp. 192-218). Champaign, IL: Human Kinetics.

Vaeyens, R., Lenoir, M., Williams, M. \& Philippaerts, R. (2007). Mechanisms underpinning successful decision making in skilled soccer players: an analysis of visual search behaviours. Journal of Motor Behaviour, 39, 395-408. 
Verrell, P. (2004). Improving visual awareness and decision making in club rugby players. Retrieved on December 12 , 2008 ARULevel3 www.rugby.com.au/verve/_resources/Improving_Visual_Awerness_- _P_Verrell_2004.pdf.

Vickers, J.N. (2007). Perception, Cognition and Decision Training: The Quiet Eye in Action. Champaign, IL: Human Kinetics. 